\title{
ANZTLA 2010 Statistics
}

\author{
by Kerrie Stevens \\ ANZTLA Statistician
}

TABLE 12010 UBRARY INFORMATION

\begin{tabular}{|c|c|c|c|}
\hline $\begin{array}{l}u \\
\stackrel{M}{Q}\end{array}$ & INSTITUTION & $\begin{array}{l}\text { STATE } \\
N Z\end{array}$ & $\begin{array}{l}\text { PREDOMNANT DENOMNATIONOF } \\
\text { COUECTION }\end{array}$ \\
\hline A & Adelaide College of Divinity & SA & Well-spread over all denominations \\
\hline $\mathrm{C}$ & Alphacrucis College & NSW & Pentecostal \\
\hline $\mathrm{C}$ & Australian Catholic University & & Catholic \\
\hline A & Australian Lutheran College & SA & Lutheran \\
\hline C & Avondale College of Higher Education & NSW & Seventh-Day Adventist \\
\hline $\mathrm{B}$ & Benedictine Community of New Norcia & WA & Catholic \\
\hline $\mathrm{C}$ & Bethlehem Tertiary Institute & $\mathrm{NZ}$ & Protestant \\
\hline A & Booth College of Mission & $\mathrm{NZ}$ & Salvation Army \\
\hline A & Camden Theological Library & NSW & Protestant \\
\hline A & Carey Baptist College & $\mathrm{NZ}$ & Baptist \\
\hline $\mathrm{B}$ & Catholic Education Office & SA & Catholic \\
\hline $\mathrm{B}$ & Catholic Education Office of Western Australia & WA & Catholic \\
\hline$A$ & Catholic Theological College & VIC & Catholic \\
\hline $\mathrm{C}$ & Christian Heritage College & QLD & Well-spread over all denominations \\
\hline$A$ & Crossway College & QLD & Well-spread over all denominations \\
\hline $\mathrm{B}$ & Dominican Fathers & $\mathrm{VIC}$ & Catholic \\
\hline A & Franciscan Order of Friars Minor & VIC & Catholic \\
\hline A & Harvest Bible College & VIC & Pentecostal \\
\hline $\mathrm{A}$ & Hillsong International Leadership College & NSW & Pentecostal \\
\hline $\mathrm{B}$ & L. J. Goody Bioethics Centre & WA & Catholic \\
\hline $\mathrm{A}$ & Malyon College & QLD & Baptist \\
\hline $\mathrm{A}$ & Mary Andrews College & NSW & Anglican \\
\hline $\mathrm{C}$ & Morling College & NSW & Well-spread over all denominations \\
\hline $\mathrm{A}$ & Nazarene Theological College & QLD & Methodist \\
\hline $\mathrm{A}$ & Queensland Theological College & QLD & Presbyterian \\
\hline A & Ridley Melbourne Mission \& Ministry College & $\mathrm{VIC}$ & Anglican \\
\hline $\mathrm{B}$ & St Benedict's Monastery & NSW & Catholic \\
\hline $\mathrm{A}$ & St Francis Theological College & QLD & Anglican \\
\hline $\mathrm{A}$ & St Mark's National Theological Centre & $\mathrm{ACT}$ & Anglican \\
\hline A & Sydney Missionary and Bible College & NSW & Protestant \\
\hline $\mathrm{A}$ & Tabor College NSW & NSW & Well-spread over all denominations \\
\hline C & Tabor College Tasmania & TAS & Protestant \\
\hline $\mathrm{C}$ & Tabor Victoria & VIC & Well-spread over all denominations \\
\hline $\mathrm{A}$ & Trinity College & $\mathrm{VIC}$ & Anglican \\
\hline$A$ & Trinity Theological College & QLD & Protestant \\
\hline $\mathrm{A}$ & Vose Seminary & WA & Baptist \\
\hline $\mathrm{C}$ & Wesley Institute & NSW & Well-spread over all denominations \\
\hline$A$ & Whitley College & $\mathrm{VIC}$ & Baptist \\
\hline
\end{tabular}




\begin{tabular}{|l|c|l|c|}
\hline Anglican & 5 & Pentecostal & 3 \\
\hline Baptist & 4 & Presbyterian & 1 \\
\hline Catholic & 9 & Protestant & 5 \\
\hline & & Salvation Army & 1 \\
\hline Lutheran & 1 & Seventh Day Adventist & 1 \\
\hline Methodist & 1 & Well-spread over all denominations & 7 \\
\hline
\end{tabular}

\section{Dominant Denominations of Collections}

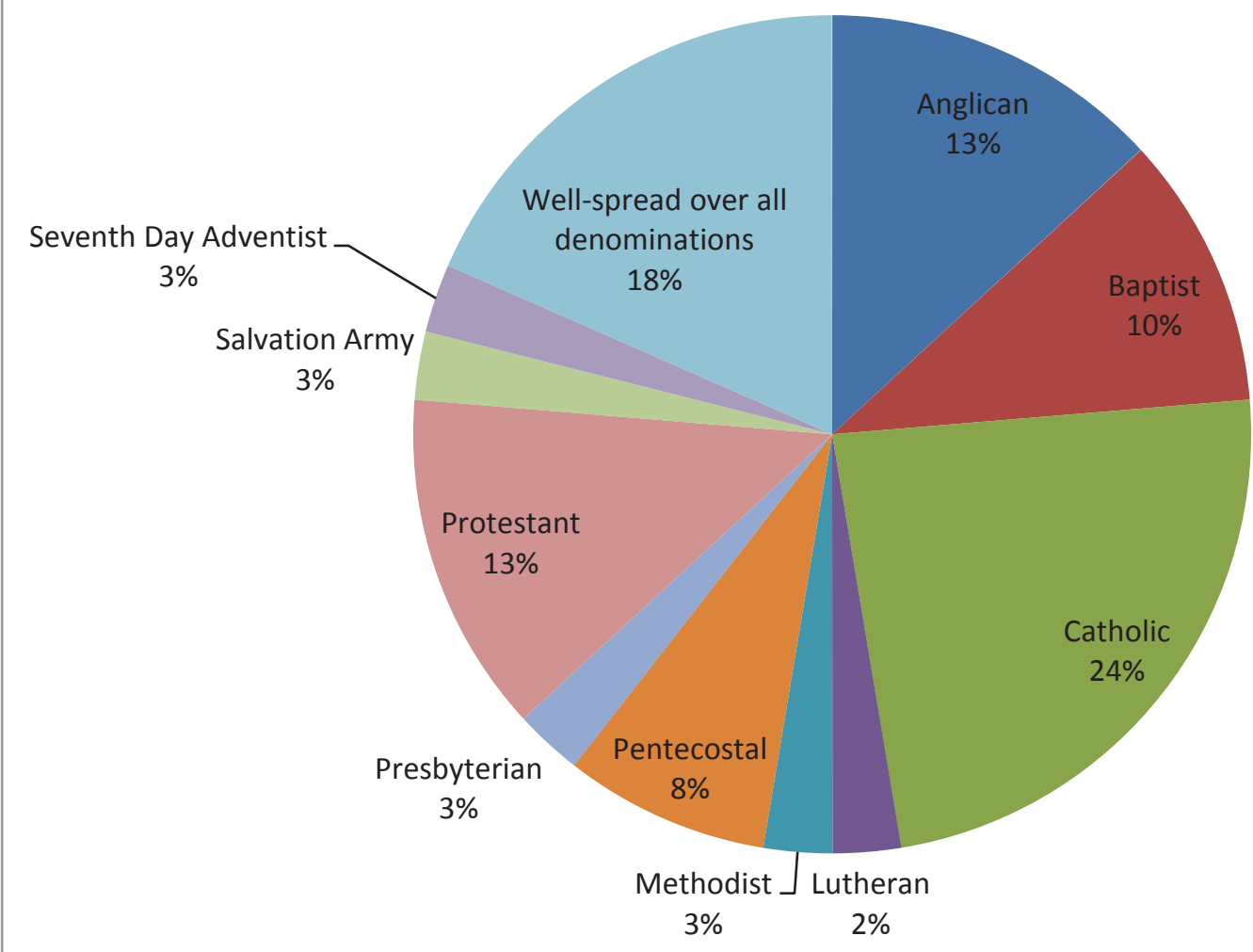


TABLE2 2010 LBRARY STAF

\begin{tabular}{|c|c|c|c|c|c|c|c|}
\hline \multirow[t]{2}{*}{$\begin{array}{l}u \\
m \\
\end{array}$} & \multirow[t]{2}{*}{ INSTITUTION } & \multirow[t]{2}{*}{$\begin{array}{c}\text { STATE } \\
\mathrm{NZ}\end{array}$} & $\begin{array}{c}\text { PROF. } \\
\text { POSITIONS }\end{array}$ & $\begin{array}{l}\text { PARA-PROF. } \\
\text { POSITIONS }\end{array}$ & $\begin{array}{l}\text { SUPPORT } \\
\text { STAFF } \\
\text { POSITIONS }\end{array}$ & VOLUNTEERS & TOTAL STAFF \\
\hline & & & [1] & [2] & [3] & [4] & [5] \\
\hline$A$ & Adelaide College of Divinity & SA & 1.7 & 0.5 & 0.08 & 0.15 & 2.43 \\
\hline $\mathrm{C}$ & Alphacrucis College & NSW & 1 & 0.4 & 0.4 & & 1.80 \\
\hline $\mathrm{C}$ & Australian Catholic University & & 40 & 26 & 15 & & 81.00 \\
\hline$A$ & Australian Lutheran College & SA & 3.4 & & 0.5 & & 3.90 \\
\hline C & Avondale College of Higher Education & NSW & 6.2 & 2.8 & 0.5 & & 9.50 \\
\hline$B$ & Benedictine Community of New Norcia & WA & 0.6 & & & 0.25 & 0.85 \\
\hline C & Bethlehem Tertiary Institute & NZ & 1 & & 0.19 & & 1.19 \\
\hline$A$ & Booth College of Mission & NZ & 1 & & & & 1.00 \\
\hline$A$ & Camden Theological Library & NSW & 1 & & 2 & & 3.00 \\
\hline $\mathrm{A}$ & Carey Baptist College & NZ & 3 & & & & 3.00 \\
\hline$B$ & Catholic Education Office & SA & 1 & & 1 & 0.4 & 2.40 \\
\hline $\mathrm{B}$ & Catholic Education Office of Western & WA & 3.5 & 1.5 & 3 & & 8.00 \\
\hline$A$ & Catholic Theological College & $\mathrm{VIC}$ & 1.8 & 1 & 0.3 & & 3.10 \\
\hline C & Christian Heritage College & QLD & 2 & & 1.9 & & 3.90 \\
\hline$A$ & Crossway College & QLD & 0.6 & & 0.1 & 0.45 & 1.15 \\
\hline$B$ & Dominican Fathers & $\mathrm{VIC}$ & 0.7 & & & 0.3 & 1.00 \\
\hline$A$ & Franciscan Order of Friars Minor & $\mathrm{VIC}$ & 2 & & 0.6 & & 2.60 \\
\hline$A$ & Harvest Bible College & VIC & 1 & & & & 1.00 \\
\hline A & Hillsong International Leadership College & NSW & 1 & & 1 & & 2.00 \\
\hline$B$ & L. J. Goody Bioethics Centre & WA & & 0.2 & & & 0.20 \\
\hline A & Malyon College & QLD & 0.618 & & & 0.5 & 1.12 \\
\hline A & Mary Andrews College & NSW & & & 0.33 & & 0.33 \\
\hline $\mathrm{C}$ & Morling College & NSW & 1 & 0.92 & 0.37 & 0.17 & 2.46 \\
\hline A & Nazarene Theological College & QLD & 1 & & 1 & & 2.00 \\
\hline$A$ & Queensland Theological College & QLD & 1 & & & 0.4 & 1.40 \\
\hline A & Ridley Melbourne Mission \& Ministry College & VIC & 1.4 & & 0.35 & & 1.75 \\
\hline B & St Benedict's Monastery & NSW & 0.2 & & & & 0.20 \\
\hline A & St Francis Theological College & QLD & 1.4 & 0.35 & & 0.1 & 1.85 \\
\hline$A$ & St Mark's National Theological Centre & ACT & 2 & 1 & & 8 & 11.00 \\
\hline A & Sydney Missionary and Bible College & NSW & 1.8 & 1 & 0.6 & 1 & 4.40 \\
\hline A & Tabor College NSW & NSW & 0.6 & & & & 0.60 \\
\hline $\mathrm{C}$ & Tabor College Tasmania & TAS & & 0.5 & & 0.4 & 0.90 \\
\hline $\mathrm{C}$ & Tabor Victoria & $\mathrm{VIC}$ & 1.8 & & 0.4 & 0.8 & 3.00 \\
\hline$A$ & Trinity College & $\mathrm{VIC}$ & 2.1 & 1.5 & & & 3.60 \\
\hline A & Trinity Theological College & QLD & 1 & 0.5 & & & 1.50 \\
\hline$A$ & Vose Seminary & WA & 0.6 & 0.6 & & 0.8 & 2.00 \\
\hline $\mathrm{C}$ & Wesley Institute & NSW & 1.8 & 0.8 & 0.5 & & 3.10 \\
\hline$A$ & Whitley College & $\mathrm{VIC}$ & 0.8 & 0.18 & 0.42 & & 1.40 \\
\hline TOT & & & 91.618 & 39.75 & 30.54 & 13.72 & 175.628 \\
\hline$\overline{A V E}$ & RAGE & & 2.6176571 & 2.3382353 & 1.3881818 & 0.98 & 4.6217895 \\
\hline
\end{tabular}


TABLE 32010 UBRARY SERMCES

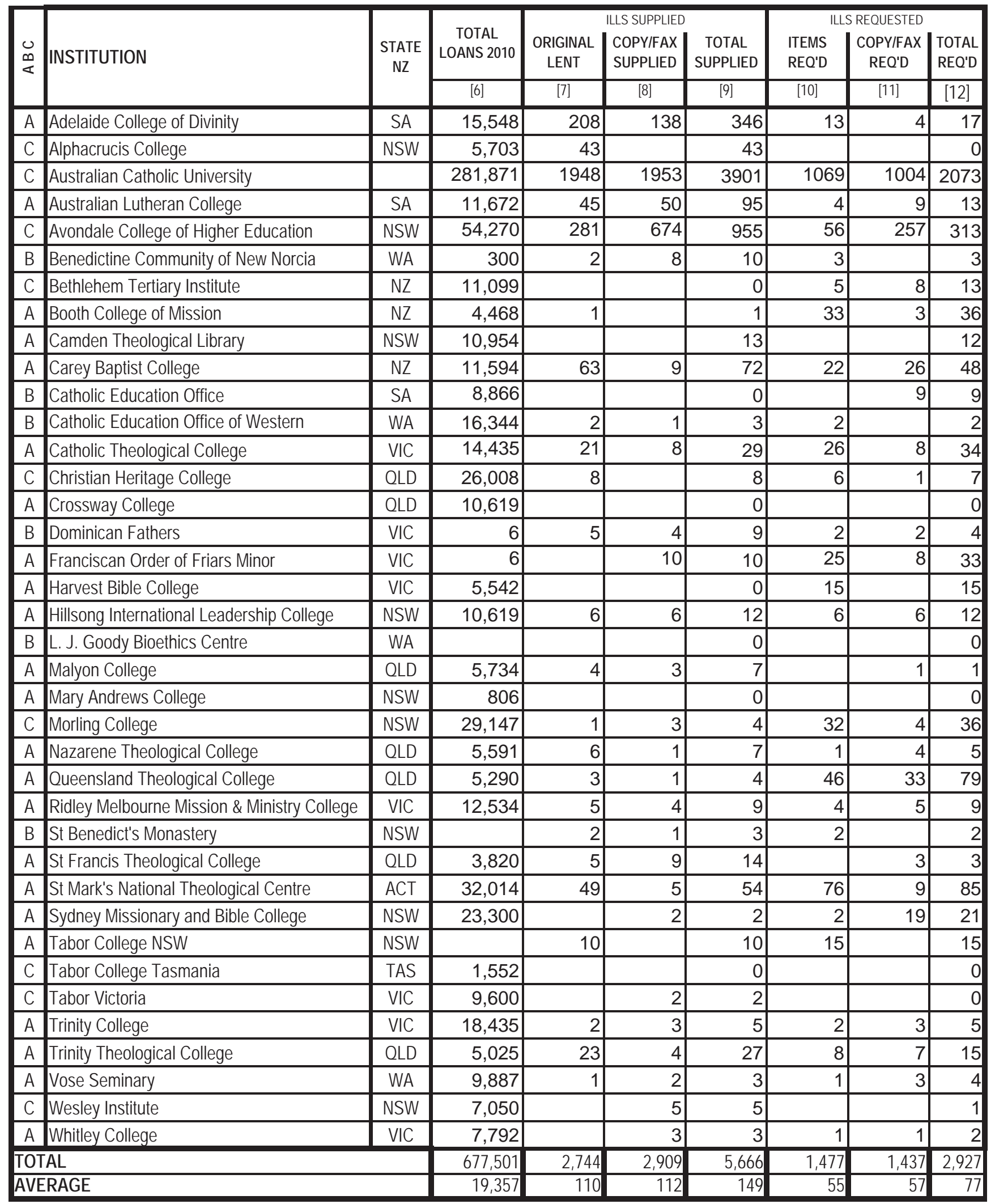

* Whole of college statistics 
TABLE 42010 LOAN PERIODS

\begin{tabular}{|c|c|c|c|c|c|c|c|}
\hline \multirow[t]{2}{*}{$\begin{array}{l}U \\
m \\
\end{array}$} & \multirow[t]{2}{*}{ INSTITUTION } & \multirow[t]{2}{*}{$\begin{array}{c}\text { STATE } \\
\mathrm{NZ}\end{array}$} & $\begin{array}{l}\text { UNDER- } \\
\text { GRADS }\end{array}$ & POST-GRADS & $\begin{array}{l}\text { LOAN PERIODS } \\
\text { ACADEMC } \\
\text { STAFF }\end{array}$ & $\begin{array}{l}\text { NON-AC. } \\
\text { STAFF }\end{array}$ & PUBபC \\
\hline & & & [13] & [14] & [15] & {$[16]$} & [17] \\
\hline A & Adelaide College of Divinity & SA & 4 weeks & 4 weeks & 4 weeks & 4 weeks & 4 weeks \\
\hline$C^{*}$ & Alphacrucis College & NSW & 2 weeks & 2 weeks & 2 weeks & 2 weeks & $\mathrm{n} / \mathrm{a}$ \\
\hline C & Australian Catholic University & & 2 weeks & 4 weeks & 4 weeks & 4 weeks & 2 weeks \\
\hline$A^{*}$ & Australian Lutheran College & $\mathrm{SA}$ & 1 week & 1 week & 1 week & 1 week & 1 week \\
\hline C & Avondale College of Higher Education & NSW & 2 weeks & 4 weeks & 12 weeks & 12 weeks & 2 weeks \\
\hline $\mathrm{B}$ & Benedictine Community of New Norcia & WA & & & & & \\
\hline $\mathrm{C}$ & Bethlehem Tertiary Institute & NZ & 2 weeks & $\mathrm{n} / \mathrm{a}$ & 12 weeks & 4 weeks & $\mathrm{n} / \mathrm{a}$ \\
\hline$A$ & Booth College of Mission & $\mathrm{NZ}$ & 6 weeks & 6 weeks & 6 weeks & 6 weeks & 6 weeks \\
\hline A & Camden Theological Library & NSW & 3 weeks & 6 weeks & 6 weeks & 3 weeks & 3 weeks \\
\hline$A$ & Carey Baptist College & NZ & 4 weeks & 4 weeks & 4 weeks & 4 weeks & 4 weeks \\
\hline $\mathrm{B}$ & Catholic Education Office & SA & 4 weeks & 4 weeks & 4 weeks & 4 weeks & 4 weeks \\
\hline$B$ & Catholic Education Office of Western Australia & WA & 4 weeks & 4 weeks & 4 weeks & 4 weeks & 4 weeks \\
\hline A & Catholic Theological College & $\mathrm{VIC}$ & 2 weeks & 2 weeks & 4 weeks & 4 weeks & 2 weeks \\
\hline C & Christian Heritage College & QLD & 2 weeks & 2 weeks & 1 semester & 2 weeks & 2 weeks \\
\hline A & Crossway College & QLD & 1 week & 3 weeks & 100 days & 100 days & 1 week \\
\hline B & Dominican Fathers & $\mathrm{VIC}$ & 4 weeks & 4 weeks & 4 weeks & 4 weeks & 4 weeks \\
\hline A & Franciscan Order of Friars Minor & $\mathrm{VIC}$ & 2 weeks & 4 weeks & 6 months & 4 weeks & 2 weeks \\
\hline$A$ & Harvest Bible College & $\mathrm{VIC}$ & 2 weeks & 4 weeks & 4 weeks & 4 weeks & 2 weeks \\
\hline A & Hillsong International Leadership College & NSW & 2 weeks & $\mathrm{n} / \mathrm{a}$ & 6 weeks & 2 weeks & n/a \\
\hline B & L. J. Goody Bioethics Centre & WA & 2 weeks & 2 weeks & 5 weeks & 2 weeks & $\mathrm{n} / \mathrm{a}$ \\
\hline A & Malyon College & QLD & 2 weeks & 4 weeks & 120 days & 2 weeks & 2 weeks \\
\hline A & Mary Andrews College & NSW & 2 weeks & 2 weeks & 2 weeks & 2 weeks & 2 weeks \\
\hline $\mathrm{C}$ & Morling College & NSW & 2 weeks & 2 weeks & 12 weeks & 4 weeks & 2 weeks \\
\hline A & Nazarene Theological College & QLD & 10 days & 4 weeks & 4 weeks & 2 weeks & 2 weeks \\
\hline$A$ & Queensland Theological College & QLD & 1 week & 1 week & 4 weeks & 1 week & 1 week \\
\hline$A$ & Ridley Melbourne Mission \& Ministry College & VIC & 2 weeks & to end of $\mathrm{yr}$ & to end of $\mathrm{yr}$ & to end of $y r$ & 2 weeks \\
\hline $\mathrm{B}$ & St Benedict's Monastery & NSW & $\mathrm{n} / \mathrm{a}$ & $\mathrm{n} / \mathrm{a}$ & $\mathrm{n} / \mathrm{a}$ & $\mathrm{n} / \mathrm{a}$ & $a$ \\
\hline $\mathrm{A}$ & St Francis Theological College & QLD & 2 weeks & 4 weeks & 4 weeks & 2 weeks & 2 weeks \\
\hline $\mathrm{A}$ & St Mark's National Theological Centre & ACT & 4 weeks & 4 weeks & 4 weeks & 2 weeks & 2 weeks \\
\hline$A$ & Sydney Missionary and Bible College & NSW & 1 week & 3 weeks & to end of $\mathrm{yr}$ & to end of $\mathrm{yr}$ & $\mathrm{n} / \mathrm{a}$ \\
\hline A & Tabor College NSW & NSW & 2 weeks & 4 weeks & 4 weeks & 2 weeks & 2 weeks \\
\hline C & Tabor College Tasmania & TAS & 3 weeks & 3 weeks & 4 weeks & 4 weeks & $\mathrm{n} / \mathrm{a}$ \\
\hline C & Tabor Victoria & $\mathrm{VIC}$ & 3 weeks & 4 weeks & 8 weeks & 4 weeks & 4 weeks \\
\hline$A$ & Trinity College & $\mathrm{VIC}$ & 3 weeks & 3 weeks & 3 weeks & 3 weeks & $n / a$ \\
\hline$A$ & Trinity Theological College & QLD & 2 weeks & 4 weeks & 4 weeks & 4 weeks & 2 weeks \\
\hline$A$ & Vose Seminary & WA & 3 weeks & 6 weeks & 6 weeks & 3 weeks & 3 weeks \\
\hline C & Wesley Institute & NSW & 2 weeks & 2 weeks & & & \\
\hline $\mathrm{A}$ & Whitley College & VIC & 2 weeks & 4 weeks & 4 weeks & 4 weeks & 2 weeks \\
\hline
\end{tabular}


TABLE 52010 COUECTION RESOURCES

\begin{tabular}{|c|c|c|c|c|c|c|c|c|c|c|c|c|}
\hline \multirow{3}{*}{ 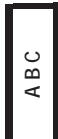 } & \multirow[t]{3}{*}{ INSTITUTION } & \multirow{3}{*}{$\begin{array}{c}\text { STATE } \\
\mathrm{NZ}\end{array}$} & \multicolumn{3}{|c|}{ MONOGRAPH VOLUMES } & \multicolumn{2}{|c|}{ NON-BOOK MATERIALS } & \multicolumn{3}{|c|}{ SERIAL VOLUMES } & \multirow{2}{*}{$\begin{array}{c}\text { ELEC. } \\
\text { SERIALS }\end{array}$} & \multirow{2}{*}{$\begin{array}{c}\text { EEECT. } \\
\text { D'BASES }\end{array}$} \\
\hline & & & ADDED 2010 & $\begin{array}{c}\text { DONATED } \\
2010\end{array}$ & $\begin{array}{c}\text { TOTALEND } \\
2010\end{array}$ & ADDED 2010 & $\begin{array}{c}\text { TOTAL END } \\
2010\end{array}$ & $\begin{array}{l}\text { ADDED } \\
2010\end{array}$ & $\begin{array}{c}\text { CURRENT } \\
\text { SUBS }\end{array}$ & $\begin{array}{c}\text { TOTAL } \\
\text { END 2010 }\end{array}$ & & \\
\hline & & & [18] & [19] & [20] & [21] & [22] & [23] & [24] & [25] & [26] & [27] \\
\hline A & Adelaide College of Divinity & SA & 1,096 & 580 & 66,100 & 10 & 165 & 6 & 197 & 560 & 1 & 8 \\
\hline $\mathrm{C}$ & Alphacrucis College & NSW & & & & & & & & & & \\
\hline $\mathrm{C}$ & Australian Catholic University & & 9,073 & 100 & 413,758 & 2,500 & 24,432 & 7 & 574 & 3,000 & 8,039 & 4,887 \\
\hline $\mathrm{A}$ & Australian Lutheran College & SA & 1,136 & 115 & 88,733 & 71 & 1,412 & & 238 & 381 & & 5 \\
\hline $\mathrm{C}$ & Avondale College of Higher Education & NSW & 3,279 & 896 & 141,742 & & & & 744 & & & 69 \\
\hline $\mathrm{B}$ & Benedictine Community of New Norcia & WA & 479 & 800 & 77,440 & 5 & 485 & & 120 & 600 & 1 & \\
\hline $\mathrm{C}$ & Bethlehem Tertiary Institute & NZ & 1,160 & 124 & 12,620 & 79 & 790 & & 65 & 154 & & 3 \\
\hline A & Booth College of Mission & NZ & 537 & & 13,238 & 51 & 1,992 & 2 & 46 & 109 & & 2 \\
\hline A & Camden Theological Library & NSW & 1,262 & & 68,798 & & & & & 500 & & 6 \\
\hline A & Carey Baptist College & NZ & 1,520 & 50 & 36,680 & 36 & 226 & & 220 & & 1 & 7 \\
\hline B & Catholic Education Office & SA & 864 & 7 & 16,220 & 80 & 2,757 & & 37 & 44 & 6 & 1 \\
\hline $\mathrm{B}$ & Catholic Education Office of Western & WA & 256 & 732 & 10,849 & 288 & 3,139 & & 42 & 49 & 4 & 3 \\
\hline A & Catholic Theological College & VIC & 618 & 902 & 10,090 & 3 & 54 & 1 & 222 & & 5 & 4 \\
\hline $\mathrm{C}$ & Christian Heritage College & QLD & 1,053 & 249 & & 35 & 35 & & & & 2 & 3 \\
\hline A & Crossway College & QLD & 1,263 & & 23,873 & 7 & 157 & 18 & 69 & 203 & & 2 \\
\hline B & Dominican Fathers & VIC & 80 & 168 & 43,000 & 5 & 47 & & 51 & 84 & & \\
\hline A & Franciscan Order of Friars Minor & VIC & 1,184 & 50 & 55,605 & 51 & 66 & & 780 & 184 & 43 & 4 \\
\hline A & Harvest Bible College & $\mathrm{VIC}$ & & & 38,955 & & 997 & 1 & 56 & 113 & & 3 \\
\hline A & Hillsong International Leadership College & NSW & 1,171 & & 16,133 & 91 & 91 & & 3 & 3 & & \\
\hline B & L. J. Goody Bioethics Centre & WA & & & & & & & & & & \\
\hline A & Malyon College & QLD & 758 & & 21,321 & 15 & 1,128 & 7 & 55 & 203 & 5 & 3 \\
\hline A & Mary Andrews College & NSW & 504 & 2 & 5,000 & 31 & 77 & 2 & 19 & 21 & & \\
\hline $\mathrm{C}$ & Morling College & NSW & 1,011 & 166 & 49,500 & 34 & 430 & 5 & 165 & 452 & & 6 \\
\hline A & Nazarene Theological College & QLD & 1,188 & 300 & 28,792 & 33 & 449 & & 26 & 100 & & 1 \\
\hline A & Queensland Theological College & QLD & 795 & 164 & 15,781 & 4 & 81 & 3 & 20 & 99 & 3 & 4 \\
\hline A & Ridley Melbourne Mission \& Ministry College & $\mathrm{VIC}$ & 1,955 & & 49,491 & 9 & 792 & 7 & 157 & 403 & & 5 \\
\hline B & St Benedict's Monastery & NSW & 103 & 673 & 19,230 & 15 & 124 & 1 & 60 & 145 & & \\
\hline A & St Francis Theological College & QLD & 941 & 213 & 20,079 & 40 & 144 & & 75 & 112 & 1 & \\
\hline $\mathrm{A}$ & St Mark's National Theological Centre & ACT & 698 & 1,998 & 58,329 & 71 & 196 & 31 & 119 & 367 & & 2 \\
\hline A & Sydney Missionary and Bible College & NSW & 825 & 415 & 35,694 & 16 & & 5 & 144 & 248 & 22 & 4 \\
\hline A & Tabor College NSW & NSW & & & 29,390 & & 1,807 & & & 250 & 1 & 4 \\
\hline $\mathrm{C}$ & Tabor College Tasmania & TAS & 620 & & & 12 & & & & & & \\
\hline $\mathrm{C}$ & Tabor Victoria & VIC & 474 & 981 & 69,860 & 3 & & 1,191 & 4 & 116 & & 4 \\
\hline A & Trinity College & $\mathrm{VIC}$ & 3,001 & 350 & 3,351 & 212 & & & & & & 3 \\
\hline A & Trinity Theological College & QLD & 716 & 179 & 24,387 & 14 & 156 & & 45 & 119 & 3 & 1 \\
\hline $\mathrm{A}$ & Vose Seminary & WA & 1,062 & 400 & 37,198 & 62 & 1,193 & & 80 & 302 & & 3 \\
\hline $\mathrm{C}$ & Wesley Institute & NSW & 861 & 214 & & 556 & 6,502 & 5 & 107 & 458 & 11 & 9 \\
\hline$A$ & Whitley College & $\mathrm{VIC}$ & 450 & 250 & 34,146 & 10 & 184 & 2 & 126 & 340 & & 4 \\
\hline TOT & $A L$ & & 41,993 & 11,078 & $1,635,383$ & 4,449 & 50,108 & 1,294 & 4,666 & 9,719 & 8,148 & 5,060 \\
\hline AVE & RAGE & & 1,235 & 410 & 49,557 & 139 & 1,670 & 76 & 151 & 324 & 509 & 174 \\
\hline
\end{tabular}

\footnotetext{
* Whole of college statistics
} 
TABLE 62010 UBRARY EXPENDITURE

\begin{tabular}{|c|c|c|c|c|c|c|c|c|}
\hline \multirow{2}{*}{ 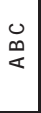 } & \multirow[t]{2}{*}{ INSTITUTION } & \multirow{2}{*}{$\begin{array}{c}\text { STATE } / N \\
z\end{array}$} & MONOGRAPHS & $\begin{array}{l}\text { NON-BOOK } \\
\text { MATERIALS }\end{array}$ & SERIAL SUBS. & $\begin{array}{c}\text { EECTRONIC SERIAL } \\
\text { SUBS. }\end{array}$ & $\begin{array}{c}\text { EECCTRONIC } \\
\text { DATABASE SUBS. }\end{array}$ & TOTAL SPENT \\
\hline & & & [28] & [29] & [30] & [31] & [32] & [33] \\
\hline A & Adelaide College of Divinity & SA & \multicolumn{2}{|c|}{$\$ 42,765$} & \multicolumn{2}{|c|}{$\$ 28,773$} & $\$ 7,379$ & $\$ 78,917$ \\
\hline C & Alphacrucis College & NSW & & & & & & \\
\hline $\mathrm{C}$ & Australian Catholic University & & \multicolumn{2}{|c|}{$\$ 1,428,892$} & $\$ 99,891$ & $\$ 561,625$ & $\$ 388,180$ & $\$ 2,478,678$ \\
\hline $\mathrm{A}$ & Australian Lutheran College & SA & $\$ 43,500$ & $\$ 3,000$ & $\$ 23,000$ & & $\$ 7,326$ & $\$ 76,826$ \\
\hline $\mathrm{C}$ & Avondale College of Higher Education & NSW & \multicolumn{2}{|c|}{$\$ 179,045$} & $\$ 155,779$ & \multicolumn{2}{|c|}{$\$ 141,579$} & $\$ 476,403$ \\
\hline$B$ & Benedictine Community of New Norcia & WA & $\$ 8,248$ & $\$ 50$ & $\$ 2,097$ & & & $\$ 11,395$ \\
\hline $\mathrm{C}$ & Bethlehem Tertiary Institute & $\mathrm{NZ}$ & \multicolumn{2}{|c|}{$\$ 24,147$} & $\$ 11,464$ & & $\$ 14,718$ & $\$ 50,329$ \\
\hline $\mathrm{A}$ & Booth College of Mission & NZ & $\$ 9,519$ & $\$ 810$ & $\$ 2,621$ & & $\$ 1,300$ & $\$ 14,250$ \\
\hline A & Camden Theological Library & NSW & $\$ 61,650$ & & $\$ 9,270$ & & $\$ 11,330$ & $\$ 82,250$ \\
\hline A & Carey Baptist College & NZ & $\$ 45,000$ & & $\$ 12,500$ & & $\$ 11,000$ & $\$ 68,500$ \\
\hline $\mathrm{B}$ & Catholic Education Office & SA & & & & & & $\$ 25,000$ \\
\hline $\mathrm{B}$ & Catholic Education Office of Western Australia & WA & $\$ 9,168$ & $\$ 12,957$ & $\$ 3,497$ & $\$ 7,787$ & $\$ 24,575$ & $\$ 57,984$ \\
\hline$A$ & Catholic Theological College & VIC & $\$ 38,300$ & & $\$ 29,950$ & & $\$ 4,565$ & $\$ 72,815$ \\
\hline $\mathrm{C}$ & Christian Heritage College & QLD & $\$ 47,255$ & $\$ 3,665$ & $\$ 1,200$ & & $\$ 8,453$ & $\$ 60,573$ \\
\hline$A$ & Crossway College & QLD & $\$ 25,621$ & $\$ 58$ & $\$ 4,219$ & & $\$ 1,448$ & $\$ 31,346$ \\
\hline $\mathrm{B}$ & Dominican Fathers & VIC & & & & & & \\
\hline$A$ & Franciscan Order of Friars Minor & VIC & $\$ 35,000$ & & $\$ 8,900$ & & $\$ 4,500$ & $\$ 44,000$ \\
\hline$A$ & Harvest Bible College & VIC & & & & & & $\$ 25,898$ \\
\hline$A$ & Hillsong International Leadership College & NSW & $\$ 9,948$ & $\$ 100$ & & & & $\$ 10,048$ \\
\hline $\mathrm{B}$ & L. J. Goody Bioethics Centre & WA & & & & & & \\
\hline $\mathrm{A}$ & Malyon College & QLD & $\$ 29,972$ & & $\$ 5,026$ & & $\$ 6,442$ & $\$ 41,440$ \\
\hline A & Mary Andrews College & NSW & \multicolumn{2}{|c|}{$\$ 13,264$} & $\$ 750$ & & & $\$ 14,014$ \\
\hline $\mathrm{C}$ & Morling College & NSW & $\$ 33,400$ & & $\$ 11,800$ & & $\$ 13,400$ & $\$ 58,600$ \\
\hline $\mathrm{A}$ & Nazarene Theological College & QLD & $\$ 4,000$ & $\$ 1,335$ & $\$ 2,345$ & & $\$ 1,667$ & $\$ 7,237$ \\
\hline$A$ & Queensland Theological College & QLD & $\$ 28,694$ & $\$ 4,165$ & $\$ 846$ & $\$ 810$ & $\$ 8,549$ & $\$ 43,064$ \\
\hline $\mathrm{A}$ & Ridley Melbourne Mission \& Ministry College & $\mathrm{VIC}$ & \multicolumn{2}{|c|}{$\$ 73,350$} & $\$ 11,243$ & & $\$ 8,288$ & $\$ 92,881$ \\
\hline $\mathrm{B}$ & St Benedict's Monastery & NSW & $\$ 8,127$ & & $\$ 4,757$ & & & $\$ 12,884$ \\
\hline $\mathrm{A}$ & St Francis Theological College & QLD & $\$ 20,075$ & $\$ 4,459$ & $\$ 5,972$ & & $\$ 2,799$ & $\$ 28,845$ \\
\hline A & St Mark's National Theological Centre & ACT & $\$ 31,896$ & $\$ 669$ & $\$ 10,746$ & & $\$ 3,538$ & $\$ 46,180$ \\
\hline A & Sydney Missionary and Bible College & NSW & $\$ 35,988$ & $\$ 220$ & $\$ 6,897$ & $\$ 7,167$ & $\$ 8,219$ & $\$ 58,491$ \\
\hline A & Tabor College NSW & NSW & & & & & & \\
\hline $\mathrm{C}$ & Tabor College Tasmania & TAS & & & & & & $\$ 3,000$ \\
\hline $\mathrm{C}$ & Tabor Victoria & $\mathrm{VIC}$ & $\$ 18,114$ & $\$ 191$ & $\$ 16,207$ & & $\$ 12,272$ & $\$ 46,784$ \\
\hline A & Trinity College & $\mathrm{VIC}$ & & & & & $\$ 10,609$ & \\
\hline A & Trinity Theological College & QLD & $\$ 31,500$ & $\$ 417$ & $\$ 5,278$ & $\$ 359$ & $\$ 900$ & $\$ 38,454$ \\
\hline A & Vose Seminary & WA & $\$ 23,800$ & $\$ 1,200$ & $\$ 8,000$ & & $\$ 7,000$ & $\$ 40,000$ \\
\hline $\mathrm{C}$ & Wesley Institute & NSW & $\$ 30,576$ & $\$ 2,759$ & $\$ 4,783$ & & $\$ 14,666$ & $\$ 52,783$ \\
\hline A & Whitley College & VIC & $\$ 25,500$ & $\$ 2,400$ & $\$ 12,980$ & & $\$ 10,400$ & $\$ 51,280$ \\
\hline TOT & $\Gamma A L$ & & $\$ 2,416,315$ & $\$ 38,454$ & $\$ 500,791$ & $\$ 719,327$ & $\$ 593,522$ & $\$ 4,301,149$ \\
\hline AVE & BAGE & & $\$ 80,544$ & $\$ 2,262$ & $\$ 17,269$ & $\$ 119,888$ & $\$ 22,828$ & $\$ 130,338$ \\
\hline
\end{tabular}

* Whole of college statistics 
TABLE 72010 INSTTTUTONAL POPULATION

\begin{tabular}{|c|c|c|c|c|c|c|c|c|c|c|}
\hline \multirow{3}{*}{ 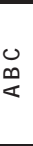 } & \multirow{3}{*}{ INSTITUTION } & \multirow{3}{*}{$\begin{array}{c}\text { STATE } / N \\
z\end{array}$} & \multicolumn{2}{|c|}{ ACADEMCSTAFF } & \multicolumn{2}{|c|}{ NON-ACADEMCSTAF } & \multicolumn{3}{|c|}{ STUDENT POPULATION } & \multirow[b]{2}{*}{$\begin{array}{l}\text { REGISTERED } \\
\text { BORROWERS }\end{array}$} \\
\hline & & & PULTIME & PART TIME & FULTINE & PART TIME & FUL TIME & | PART TIME & ET & \\
\hline & & & [34] & [35] & [36] & {$[37]$} & [38] & [39] & [40] & [41] \\
\hline $\mathrm{A}$ & Adelaide College of Divinity & SA & 25 & 65 & 10 & 9 & & & 110 & 148 \\
\hline $\mathrm{C}$ & Alphacrucis College & NSW & & & & & & & & \\
\hline $\mathrm{C}$ & Australian Catholic University & & 438 & 73 & 520 & 123 & 12,917 & 7,126 & 14,843 & 306 \\
\hline A & Australian Lutheran College & SA & 14 & 4 & 23 & 8 & 51 & 263 & 93 & 63 \\
\hline C & Avondale College of Higher Education & NSW & & & & & & & & \\
\hline $\mathrm{B}$ & Benedictine Community of New Norcia & WA & & & & & & & & 12 \\
\hline C & Bethlehem Tertiary Institute & NZ & & & & & & & & \\
\hline $\mathrm{A}$ & Booth College of Mission & $\mathrm{NZ}$ & 12 & 1 & 6 & 2 & 34 & 6 & 30 & 336 \\
\hline A & Camden Theological Library & NSW & 5 & 0 & 1 & 5 & 101 & & & \\
\hline A & Carey Baptist College & $\mathrm{NZ}$ & 7 & 10 & 13 & 5 & 140 & 280 & 167 & 12 \\
\hline $\mathrm{B}$ & Catholic Education Office & SA & & & & & & 1,697 & & 5,323 \\
\hline $\mathrm{B}$ & Catholic Education Office of Western Australia & WA & & & & & & & & 5,726 \\
\hline A & Catholic Theological College & $\mathrm{VIC}$ & 6 & 36 & 4 & 1 & 69 & 348 & 137 & 226 \\
\hline $\mathrm{C}$ & Christian Heritage College & QLD & & & & & & & & \\
\hline A & Crossway College & QLD & 5 & 7 & 4 & & 42 & 42 & 52 & 3 \\
\hline $\mathrm{B}$ & Dominican Fathers & $\mathrm{VIC}$ & & 3 & & 1 & 5 & 1 & 5 & 370 \\
\hline A & Franciscan Order of Friars Minor & $\mathrm{VIC}$ & 6 & 30 & 1 & 4 & & & 150 & 50 \\
\hline A & Harvest Bible College & $\mathrm{VIC}$ & 6 & 1 & 3 & 4 & & & 260 & 402 \\
\hline A & Hillsong International Leadership College & NSW & 25 & 10 & 20 & 3 & 732 & & & 825 \\
\hline $\mathrm{B}$ & L. J. Goody Bioethics Centre & WA & 2 & & 1 & 1 & & & & \\
\hline A & Malyon College & QLD & 4 & 4 & 2 & 2 & 46 & 104 & 84 & 81 \\
\hline A & Mary Andrews College & NSW & 1 & 6 & 1 & 3 & 2 & 136 & 52 & \\
\hline C & Morling College & NSW & & & & & & & & \\
\hline $\mathrm{A}$ & Nazarene Theological College & QLD & 4 & 3 & 1 & 7 & 75 & 23 & 10 & 108 \\
\hline $\mathrm{A}$ & Queensland Theological College & QLD & 4 & 3 & 1 & 4 & 54 & 26 & 57 & 223 \\
\hline A & Ridley Melbourne Mission \& Ministry College & $\mathrm{VIC}$ & 7 & 20 & 2 & 14 & 65 & 183 & 120 & 371 \\
\hline $\mathrm{B}$ & St Benedict's Monastery & NSW & 10 & & & & & & & \\
\hline A & St Francis Theological College & QLD & 3 & 6 & 1 & 3 & 13 & 43 & 33 & 208 \\
\hline $\mathrm{A}$ & St Mark's National Theological Centre & $\mathrm{ACT}$ & 5 & 29 & 2 & 6 & 15 & 325 & 122 & 314 \\
\hline A & Sydney Missionary and Bible College & NSW & 20 & 28 & 17 & 10 & 217 & 255 & 288 & 547 \\
\hline $\mathrm{A}$ & Tabor College NSW & NSW & & & & & & & & 113 \\
\hline $\mathrm{C}$ & Tabor College Tasmania & TAS & & & & & & & & \\
\hline $\mathrm{C}$ & Tabor Victoria & $\mathrm{VIC}$ & & & & & & & & \\
\hline $\mathrm{A}$ & Trinity College & $\mathrm{VIC}$ & 8 & 13 & 1 & & & 46 & & \\
\hline A & Trinity Theological College & QLD & 6 & 4 & 1 & 2 & 18 & 21 & & 230 \\
\hline A & Vose Seminary & WA & 3 & 12 & 5 & 3 & & & 100 & 98 \\
\hline $\mathrm{C}$ & Wesley Institute & NSW & & & & & & & & \\
\hline & Whitley College & $\mathrm{VIC}$ & 7 & 45 & 6 & 6 & & & 130 & 78 \\
\hline & TAL & & 633 & 413 & 646 & 225.7 & 14596 & 10626 & 16843.1 & 16173 \\
\hline & ERAGE & & 25.32 & 17.208333 & 26.916667 & 9.8130435 & 810.88889 & 708.4] & 842.155 & 646.92 \\
\hline
\end{tabular}

* Whole of college statistics 
TABLE 82010 BIBUOGRAPHIC INSTRUCTION

\begin{tabular}{|c|c|c|c|c|}
\hline \multirow{3}{*}{ 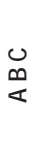 } & \multirow{3}{*}{ INSTITUTION } & \multirow{3}{*}{$\begin{array}{c}\text { STATE/N } \\
Z\end{array}$} & \multicolumn{2}{|c|}{ BIBLIOGRAPHIC INSTRUCTION } \\
\hline & & & $\begin{array}{c}\text { NO. OF } \\
\text { ATTENDEES }\end{array}$ & $\begin{array}{c}\text { NO. OF } \\
\text { SESSIONS }\end{array}$ \\
\hline & & & [42] & [43] \\
\hline A & Adelaide College of Divinity & SA & 78 & 8 \\
\hline C & Alphacrucis College & NSW & 40 & 2 \\
\hline C & Australian Catholic University & & 13741 & 524 \\
\hline A & Australian Lutheran College & SA & 64 & 9 \\
\hline C & Avondale College of Higher Education & NSW & 1163 & 94 \\
\hline $\mathrm{B}$ & Benedictine Community of New Norcia & WA & & \\
\hline C & Bethlehem Tertiary Institute & NZ & & 8 \\
\hline $\mathrm{A}$ & Booth College of Mission & NZ & 16 & 1 \\
\hline A & Camden Theological Library & NSW & & \\
\hline A & Carey Baptist College & NZ & 100 & 14 \\
\hline $\mathrm{B}$ & Catholic Education Office & SA & 199 & 18 \\
\hline$B$ & Catholic Education Office of Western Australia & WA & 108 & 11 \\
\hline A & Catholic Theological College & VIC & & \\
\hline C & Christian Heritage College & QLD & & \\
\hline A & Crossway College & QLD & 45 & 2 \\
\hline $\mathrm{B}$ & Dominican Fathers & $\mathrm{VIC}$ & 15 & 4 \\
\hline A & Franciscan Order of Friars Minor & $\mathrm{VIC}$ & 100 & 10 \\
\hline A & Harvest Bible College & VIC & 30 & 2 \\
\hline A & Hillsong International Leadership College & NSW & & \\
\hline B & L. J. Goody Bioethics Centre & WA & & \\
\hline A & Malyon College & QLD & 30 & 2 \\
\hline A & Mary Andrews College & NSW & & \\
\hline C & Morling College & NSW & & \\
\hline A & Nazarene Theological College & QLD & 15 & 4 \\
\hline A & Queensland Theological College & QLD & 75 & 8 \\
\hline A & Ridley Melbourne Mission \& Ministry College & $\mathrm{VIC}$ & 128 & 24 \\
\hline $\mathrm{B}$ & St Benedict's Monastery & NSW & & \\
\hline A & St Francis Theological College & QLD & 44 & 4 \\
\hline A & St Mark's National Theological Centre & $\mathrm{ACT}$ & 170 & 12 \\
\hline A & Sydney Missionary and Bible College & NSW & 315 & 85 \\
\hline A & Tabor College NSW & NSW & 70 & 70 \\
\hline $\mathrm{C}$ & Tabor College Tasmania & TAS & & \\
\hline C & Tabor Victoria & $\mathrm{VIC}$ & 60 & 18 \\
\hline $\mathrm{A}$ & Trinity College & $\mathrm{VIC}$ & 40 & 3 \\
\hline A & Trinity Theological College & QLD & 27 & 2 \\
\hline A & Vose Seminary & WA & 40 & 40 \\
\hline $\mathrm{C}$ & Wesley Institute & NSW & & \\
\hline A & Whitley College & VIC & 20 & 4 \\
\hline & TAL & & 16733 & 983 \\
\hline AV & ERAGE & & 643.5769231 & 36.40740741 \\
\hline
\end{tabular}

* Whole of college statistics 
TABLE 92010 UBRARY FACIUTIES

\begin{tabular}{|c|c|c|c|c|c|c|c|}
\hline \multirow{2}{*}{ 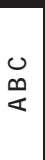 } & \multirow[t]{2}{*}{ INSTITUTION } & \multirow[t]{2}{*}{$\begin{array}{c}\text { STATEIN } \\
z\end{array}$} & $\begin{array}{l}\text { TOTAL SEATING } \\
\text { CAPACTY }\end{array}$ & $\begin{array}{c}\text { COMPUTERS } \\
\text { FOR } \\
\text { STUDENTS }\end{array}$ & $\begin{array}{l}\text { COMPUIERS } \\
\text { WTH } \\
\text { INIERNET }\end{array}$ & $\begin{array}{l}\text { MREIESS } \\
\text { AVAILABLE? }\end{array}$ & $\begin{array}{l}\text { COMPUIER } \\
\text { DOCKING } \\
\text { STATIONS }\end{array}$ \\
\hline & & & [44] & [45] & {$[46]$} & {$[47]$} & {$[48]$} \\
\hline A & Adelaide College of Divinity & SA & 40 & 8 & 12 & No & \\
\hline C & Alphacrucis College & NSW & 22 & 10 & 13 & Yes & \\
\hline C & Australian Catholic University & & 1300 & 487 & 542 & Yes & 50 \\
\hline A & Australian Lutheran College & SA & 65 & 6 & 13 & Yes & 1 \\
\hline $\mathrm{C}$ & Avondale College of Higher Education & NSW & 250 & 64 & 74 & Yes & \\
\hline$B$ & Benedictine Community of New Norcia & WA & 15 & 5 & 4 & No & \\
\hline $\mathrm{C}$ & Bethlehem Tertiary Institute & $\mathrm{NZ}$ & 26 & 9 & 11 & No & \\
\hline$A$ & Booth College of Mission & $\mathrm{NZ}$ & 53 & 4 & 4 & Yes & 10 \\
\hline A & Camden Theological Library & NSW & 30 & 11 & 16 & Yes & \\
\hline$A$ & Carey Baptist College & $\mathrm{NZ}$ & 113 & 16 & 20 & Yes & 40 \\
\hline$B$ & Catholic Education Office & SA & 20 & 3 & 5 & No & \\
\hline $\mathrm{B}$ & Catholic Education Office of Western & WA & 24 & 2 & 4 & No & \\
\hline $\bar{A}$ & Catholic Theological College & $\mathrm{VIC}$ & 72 & 11 & 15 & Yes & \\
\hline $\mathrm{C}$ & Christian Heritage College & QLD & 56 & 12 & 17 & Yes & \\
\hline A & Crossway College & QLD & 42 & 6 & 8 & Yes & 9 \\
\hline$B$ & Dominican Fathers & $\mathrm{VIC}$ & 12 & 3 & 4 & No & 1 \\
\hline$A$ & Franciscan Order of Friars Minor & VIC & 40 & 3 & 10 & Yes & \\
\hline $\mathrm{A}$ & Harvest Bible College & $\mathrm{VIC}$ & 18 & 8 & 9 & No & \\
\hline A & Hillsong International Leadership College & NSW & 45 & 41 & 44 & Yes & \\
\hline$B$ & L. J. Goody Bioethics Centre & WA & 3 & 2 & 2 & Yes & 2 \\
\hline A & Malyon College & QLD & 49 & 7 & 9 & Yes & 10 \\
\hline A & Mary Andrews College & NSW & 14 & 2 & 2 & & 2 \\
\hline $\mathrm{C}$ & Morling College & NSW & 68 & 10 & 10 & Yes & \\
\hline$A$ & Nazarene Theological College & QLD & 24 & 8 & 9 & Yes & 2 \\
\hline A & Queensland Theological College & QLD & 39 & 4 & 12 & Yes & \\
\hline$A$ & Ridley Melbourne Mission \& Ministry College & $\mathrm{VIC}$ & 80 & 8 & 11 & No & 26 \\
\hline B & St Benedict's Monastery & NSW & 10 & 1 & 2 & No & \\
\hline A & St Francis Theological College & QLD & 23 & 5 & 8 & Yes & 2 \\
\hline$A$ & St Mark's National Theological Centre & ACT & 20 & 5 & 11 & Yes & \\
\hline A & Sydney Missionary and Bible College & NSW & 90 & 6 & 11 & Yes & 3 \\
\hline A & Tabor College NSW & NSW & & 8 & 9 & Yes & \\
\hline $\mathrm{C}$ & Tabor College Tasmania & TAS & 12 & & 5 & & \\
\hline $\mathrm{C}$ & Tabor Victoria & $\mathrm{VIC}$ & 48 & 11 & 16 & Yes & 4 \\
\hline$A$ & Trinity College & $\mathrm{VIC}$ & 96 & 13 & 16 & Yes & \\
\hline A & Trinity Theological College & QLD & 32 & 6 & 9 & Yes & 6 \\
\hline $\mathrm{A}$ & Vose Seminary & WA & 33 & 5 & 10 & Yes & \\
\hline $\mathrm{C}$ & Wesley Institute & NSW & 44 & 20 & 20 & Yes & 12 \\
\hline A & Whitley College & VIC & 45 & 12 & 14 & Yes & \\
\hline & & & 2973 & 842 & 1011 & & 180 \\
\hline & ERAGE & & 80.35135135 & 22.75675676 & 26.60526316 & & 11.25 \\
\hline
\end{tabular}

* Whole of college statistics 
TABLE 102010 STAFF SALARIES

\begin{tabular}{|c|c|c|c|}
\hline INSTITUTION & PROF. & CHURCH & OTHER \\
\hline A & 10 & 4 & 7 \\
\hline B & 2 & 2 & 2 \\
\hline C & 1 & 1 & 6 \\
\hline TOTAL & 13 & 7 & 15 \\
\hline
\end{tabular}

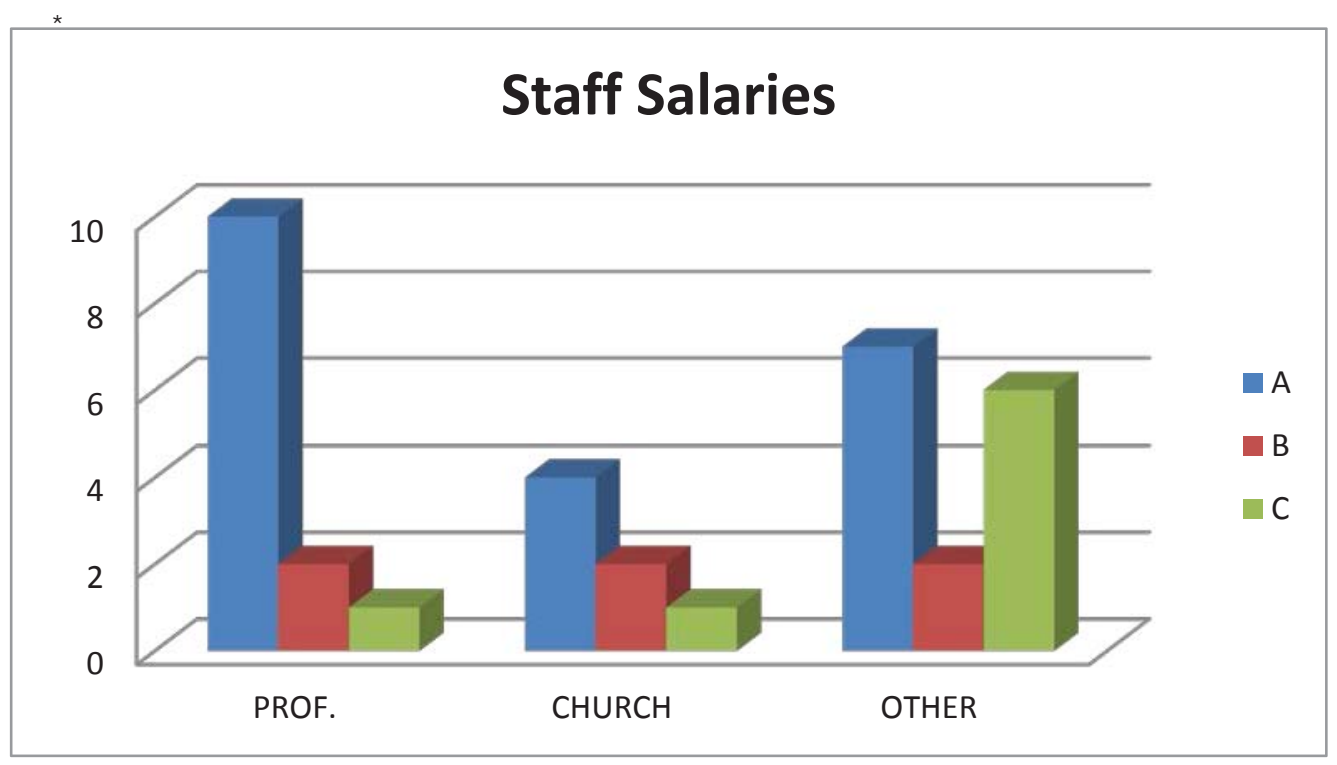


TABLE 112010 GENERAL

\begin{tabular}{|c|c|c|c|c|c|c|c|c|c|}
\hline \multirow{3}{*}{$\begin{array}{l}0 \\
\infty \\
\end{array}$} & \multirow{3}{*}{ INSTITUTION } & \multirow{3}{*}{$\begin{array}{c}\text { STATE } \\
\text { NZ }\end{array}$} & \multirow{2}{*}{$\begin{array}{l}\text { UBRARY SYSTEM } \\
\text { USED }\end{array}$} & \multicolumn{6}{|c|}{ MODULESUSED } \\
\hline & & & & ACQ. & CIRC. & SERIALS & CAT. & OPAC & OTHER \\
\hline & & & [50] & \multicolumn{6}{|c|}{ [51] } \\
\hline$A$ & Adelaide College of Divinity & SA & Voyager & & $\bar{Y}$ & & $\bar{Y}$ & $\bar{Y}$ & Reports \\
\hline $\mathrm{C}$ & Alphacrucis College & NSW & Liberty & $\bar{Y}$ & $\bar{Y}$ & $\bar{Y}$ & $\mathrm{Y}$ & $\mathrm{Y}$ & ILL \\
\hline $\mathrm{C}$ & Australian Catholic University & & Aleph & $\mathrm{Y}$ & $\mathrm{Y}$ & $\bar{Y}$ & $\bar{Y}$ & $\mathrm{Y}$ & \\
\hline$A$ & Australian Lutheran College & SA & Symphony & & $\bar{Y}$ & & $\bar{Y}$ & $\bar{Y}$ & \\
\hline C & Avondale College of Higher Education & NSW & Aleph & $\bar{Y}$ & $\bar{Y}$ & $\bar{Y}$ & $\bar{Y}$ & $\bar{Y}$ & ILL \\
\hline $\mathrm{B}$ & Benedictine Community of New Norcia & WA & Inmagic DB Text & & & & $\mathrm{Y}$ & & \\
\hline $\mathrm{C}$ & Bethlehem Tertiary Institute & $\mathrm{NZ}$ & Oliver & $\bar{Y}$ & $\mathrm{Y}$ & $\mathrm{Y}$ & $\bar{Y}$ & $\mathrm{Y}$ & \\
\hline $\mathrm{A}$ & Booth College of Mission & NZ & $\mathrm{eLm}$ & $\bar{Y}$ & $\bar{Y}$ & & $\bar{Y}$ & $\mathrm{Y}$ & \\
\hline A & Camden Theological Library & NSW & Horizon & $\mathrm{Y}$ & $\bar{Y}$ & $\mathrm{Y}$ & $\mathrm{Y}$ & $\mathrm{Y}$ & Reserve Bookroom \\
\hline$A$ & Carey Baptist College & NZ & Liberty & $\bar{Y}$ & $\bar{Y}$ & $\mathrm{Y}$ & $\mathrm{Y}$ & $\bar{Y}$ & \\
\hline $\mathrm{B}$ & Catholic Education Office & SA & AIMS Masterfile & & $\mathrm{Y}$ & $\mathrm{Y}$ & $\mathrm{Y}$ & $\mathrm{Y}$ & \\
\hline $\mathrm{B}$ & Catholic Education Office of Western Australia & WA & E-library & & $\bar{Y}$ & & $\bar{Y}$ & $\bar{Y}$ & \\
\hline $\mathrm{A}$ & Catholic Theological College & VIC & Horizon & & $\mathrm{Y}$ & & $\mathrm{Y}$ & $\mathrm{Y}$ & \\
\hline C & Christian Heritage College & QLD & Aleph & $\bar{Y}$ & $\bar{Y}$ & & $\bar{Y}$ & $\bar{Y}$ & \\
\hline$A$ & Crossway College & QLD & Destiny & $\mathrm{Y}$ & $\mathrm{Y}$ & $\mathrm{Y}$ & $\bar{Y}$ & $\mathrm{Y}$ & \\
\hline $\mathrm{B}$ & Dominican Fathers & $\mathrm{VIC}$ & Destiny & $\mathrm{Y}$ & $\mathrm{Y}$ & & $\mathrm{Y}$ & $\mathrm{Y}$ & \\
\hline $\mathrm{A}$ & Franciscan Order of Friars Minor & $\mathrm{VIC}$ & Liberty & $\mathrm{Y}$ & $\mathrm{Y}$ & $\bar{Y}$ & $\mathrm{Y}$ & $\mathrm{Y}$ & \\
\hline $\mathrm{A}$ & Harvest Bible College & $\mathrm{VIC}$ & Alice & $\bar{Y}$ & $\mathrm{Y}$ & $\mathrm{Y}$ & $\bar{Y}$ & $\bar{Y}$ & \\
\hline $\mathrm{A}$ & Hillsong International Leadership College & NSW & Liberty & $\mathrm{Y}$ & $\bar{Y}$ & $\mathrm{Y}$ & $\mathrm{Y}$ & $\mathrm{Y}$ & \\
\hline $\mathrm{B}$ & L. J. Goody Bioethics Centre & WA & Inmagic DB Text & $\bar{Y}$ & & & $\bar{Y}$ & & \\
\hline $\mathrm{A}$ & Malyon College & QLD & Liberty & $\mathrm{Y}$ & $\mathrm{Y}$ & $\bar{Y}$ & $\mathrm{Y}$ & $\mathrm{Y}$ & \\
\hline$A$ & Mary Andrews College & NSW & Filemaker Pro & & & & & & \\
\hline $\mathrm{C}$ & Morling College & NSW & Liberty & $\mathrm{Y}$ & $\mathrm{Y}$ & $\mathrm{Y}$ & $\mathrm{Y}$ & $\mathrm{Y}$ & \\
\hline $\mathrm{A}$ & Nazarene Theological College & QLD & Destiny & & & & & $\mathrm{Y}$ & \\
\hline$A$ & Queensland Theological College & QLD & Liberty & & $\mathrm{Y}$ & $\mathrm{Y}$ & $\mathrm{Y}$ & $\bar{Y}$ & Self-circulation \\
\hline $\mathrm{A}$ & Ridley Melbourne Mission \& Ministry College & $\mathrm{VIC}$ & Horizon & & $\mathrm{Y}$ & & $\mathrm{Y}$ & $\mathrm{Y}$ & \\
\hline $\mathrm{B}$ & St Benedict's Monastery & NSW & Library World & & & & $\mathrm{Y}$ & $\mathrm{Y}$ & Reports \\
\hline A & St Francis Theological College & QLD & Liberty & $\mathrm{Y}$ & $\mathrm{Y}$ & $\mathrm{Y}$ & $\mathrm{Y}$ & $\mathrm{Y}$ & \\
\hline$A$ & St Mark's National Theological Centre & $\mathrm{ACT}$ & Aleph & $\bar{Y} \mathrm{Y}$ & 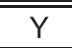 & & $\overline{\mathrm{Y}}$ & 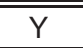 & \\
\hline $\mathrm{A}$ & Sydney Missionary and Bible College & NSW & Athena & & $\mathrm{Y}$ & $\bar{Y}$ & $\mathrm{Y}$ & $\mathrm{Y}$ & \\
\hline $\mathrm{A}$ & Tabor College NSW & NSW & Aleph & & $\mathrm{Y}$ & & $\mathrm{Y}$ & $\mathrm{Y}$ & \\
\hline $\mathrm{C}$ & Tabor College Tasmania & TAS & Bookmark & & & & & & \\
\hline C & Tabor Victoria & VIC & AIMS Masterfile & $\mathrm{Y}$ & $\mathrm{Y}$ & & $\mathrm{Y}$ & $\mathrm{Y}$ & \\
\hline$A$ & Trinity College & VIC & Symphony & $\mathrm{Y}$ & $\bar{Y}$ & $\mathrm{Y}$ & $\bar{Y}$ & $\mathrm{Y}$ & \\
\hline $\mathrm{A}$ & Trinity Theological College & QLD & Liberty & $\mathrm{Y}$ & $\mathrm{Y}$ & $\mathrm{Y}$ & $\mathrm{Y}$ & $\mathrm{Y}$ & \\
\hline $\mathrm{A}$ & Vose Seminary & WA & Liberty & & $\mathrm{Y}$ & $\mathrm{Y}$ & $\mathrm{Y}$ & $\mathrm{Y}$ & \\
\hline $\mathrm{C}$ & Wesley Institute & NSW & Alice & & $\mathrm{Y}$ & $\mathrm{Y}$ & $\mathrm{Y}$ & $\mathrm{Y}$ & \\
\hline$A$ & Whitley College & $\mathrm{VIC}$ & Liberty & $\mathrm{Y}$ & $\mathrm{Y}$ & $Y$ & $\bar{Y}$ & $\mathrm{Y}$ & \\
\hline
\end{tabular}




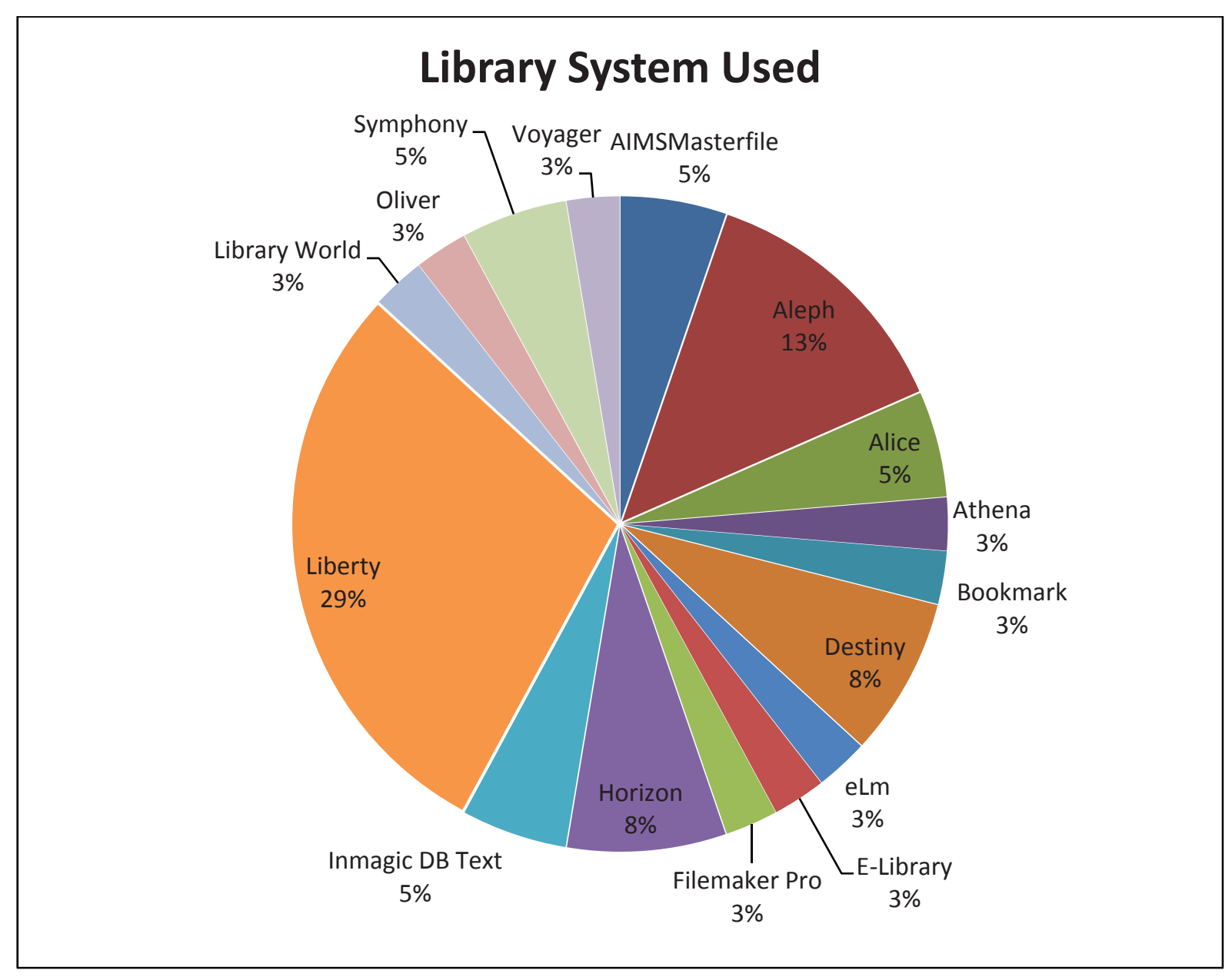

